\title{
Primary Choriocarcinoma of the Stomach. A Case Report and Review of the Literature
}

\author{
Rahul Raghavapuram $^{1} \cdot$ Fadl H Veerankutty ${ }^{2}$ M Anandakumar ${ }^{1}$
}

Received: 11 March 2015 / Accepted: 15 January 2016/Published online: 26 January 2016

(C) Indian Association of Surgical Oncology 2016

\section{Introduction}

Choriocarcinoma is a $\beta-\mathrm{HCG}$ secreting tumour which develops from trophoblastic or totipotent germ cells primarily in ovary and testis, however it can also occur rarely in extra gonadal sites like mediastinum, retroperitoneum, pineal gland, liver, gallbladder, urinary tract system etc. [1]. Choriocarcinoma can also develop from the stomach very rarely and only few cases have been reported in the literature so far [2]. Moreover, around $70 \%$ of gastric choriocarcinomas show some adenocarcinoma components along with it [3-5] and making pure choriocarcinoma of stomach a rare entity. Unlike adenocarcinoma of stomach, it is difficult to diagnose PGC by endoscopic biopsy and only 8 to $15 \%$ cases are diagnosed by it. Most cases reported were diagnosed after surgery. Here, we report a case of 60 year old female with locally advanced pure form of PGC diagnosed by endoscopic biopsy.

Key Messages Pure form of primary gastric choriocarcinoma (PGC) is very rare. Usually, it is difficult to diagnose PGC by endoscopic biopsy. We highlight the importance of early diagnosis by endoscopic biopsy and recent shift in the management of PGC from primary chemotherapy to early surgery.

Rahul Raghavapuram

rahulraghavapuram@gmail.com

1 Department of Surgical Gastroenterology, Kerala Institute of Medical Sciences, Trivandrum 695029, India

2 Department of Hepatobiliary Pancreatic and Liver Transplant Surgery, Kerala Institute of Medical Sciences, Trivandrum, India

\section{Case Report}

A 60 year old postmenopausal female reported with complaints of debility, loss of appetite with no history of abdominal pain, weight loss, hematemesis or melena. Physical examination revealed severe pallor and a palpable hard mass in the epigastrium which was $8 \times 6 \mathrm{~cm}$ in size with irregular margins and moving with respiration. There was no hepatosplenomegaly. Supraclavicular, axillary and inguinal lymph nodes were not palpable. There was no pelvic deposit on digital rectal examination. Laboratory values revealed haemoglobin level of 7.9 with normal liver function tests. Upper GI endoscopy showed a large ulceroproliferative growth extending from midpart of stomach to pylorus with luminal compromise which was biopsied (Fig. 1). As to tumour markers, AFP was elevated while CEA and CA19-9 were normal. CECT abdomen and pelvis revealed a large mass of $10 \times 6 \mathrm{~cm}$ size, arising from the distal part of stomach with luminal obstruction suggestive of carcinoma of stomach (Fig. 2a). Rest of abdominal organs were normal and there was no lymph node enlargement. Provisional diagnosis of carcinoma of stomach was made but the endoscopic biopsy revealed that lesion has typical features of choriocarcinoma. The tumour was composed of bizarre giant cells which were multi nucleated or lobulated nucleus(syncytiotrophoblasts), and oval or polygonal cells composed of clear or pale cytoplasm (Cytotrophoblasts) (Fig. 3a, b). Trophoblastic cells showed strong immunoreactivity for $\beta-\mathrm{HCG}$ (Fig. 3c) and cytokeratin and negative for CD 117, Vimentin and SMA. Serum $\beta-$ HCG was $15,600 \mathrm{mIU} / \mathrm{ml}$. HRCT chest ruled out metastasis (Fig. 1b). Finally, the diagnosis of primary gastric choriocarcinoma was established. In view of chemosensitive nature of 
Fig. 1 Upper GI endoscopy. Upper GI endoscopy picture showing a large ulceroproliferative growth from mid stomach and pylorus (arrow) with luminal compromise suggestive of carcinoma of stomach
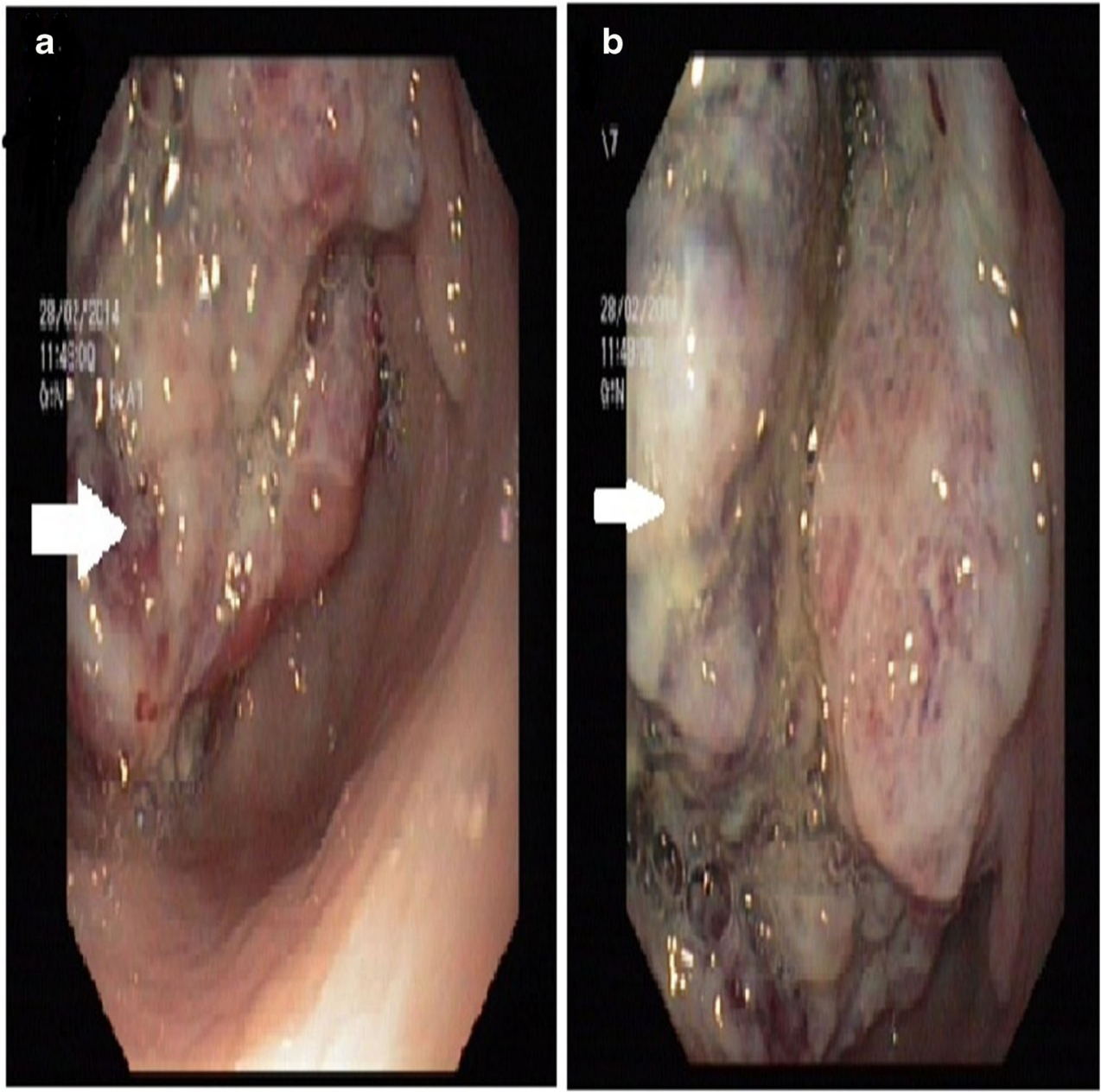

choriocarcinoma and advanced stage of the disease, patient was given 3 cycles of BEP (Bleomycin, Etoposide and Cisplatin) regimen. Post chemotherapy there was significant decline in $\beta$ - HCG levels to $380 \mathrm{mIU} / \mathrm{ml}$ and CECT abdomen showed eccentric isodense thickening of posterior wall of stomach involving midpart to pylorus measuring $1 \mathrm{~cm}$ in thickness with significant resolution in size (Fig. 2c). For clearing off residual tumour, patient was scheduled for gastrectomy. Exploration of abdomen revealed tumour arising from posterior wall of stomach with infiltration of neck and uncinate process of pancreas and root of mesentery, hence resection was abandoned and patient was subjected to further chemotherapy with Vinblastine, Ifosfomide and Cisplatin. Later patient developed pulmonary metastasis with progressive disease and expired due to respiratory failure. Her survival period was only 5 months after diagnosis.

\section{Discussion}

Choriocarcinoma of stomach is a very rare neoplasm. Kobayashi et al. performed a pooled analysis of 53 patients reported in the literature so far with PGC and described a male to female ratio of 2.3:1 with a median age at presentation of 63 years. Lower third of the stomach was found to be the most frequent location of PGC [6].

Presenting features are similar to adenocarcinoma with upper GI bleeding as the most common symptom $[1,2]$. Most cases show metastasis at the time of presentation and the most common sites of metastasis are lymph nodes followed by liver, peritoneum and lungs. In our case, there was no evidence of metastasis at presentation [6].

Several theories have been proposed regarding the mechanism of the development of PGC. Most accepted 
Fig. 2 CECT abdomen and pelvis picture showing a large mass arising from distal part of stomach and pylorus (arrow) with luminal obstruction suggestive of carcinoma of stomach. b. HRCT chest picture showing normal study with no evidence of metastasis. c. CECT abdomen post chemotherapy picture showing eccentric isodense thickening of posterior wall of stomach (arrow) involving midpart upto pylorus measuring $1 \mathrm{~cm}$ in thickness with significant resolution in size compared to prechemotherapy image
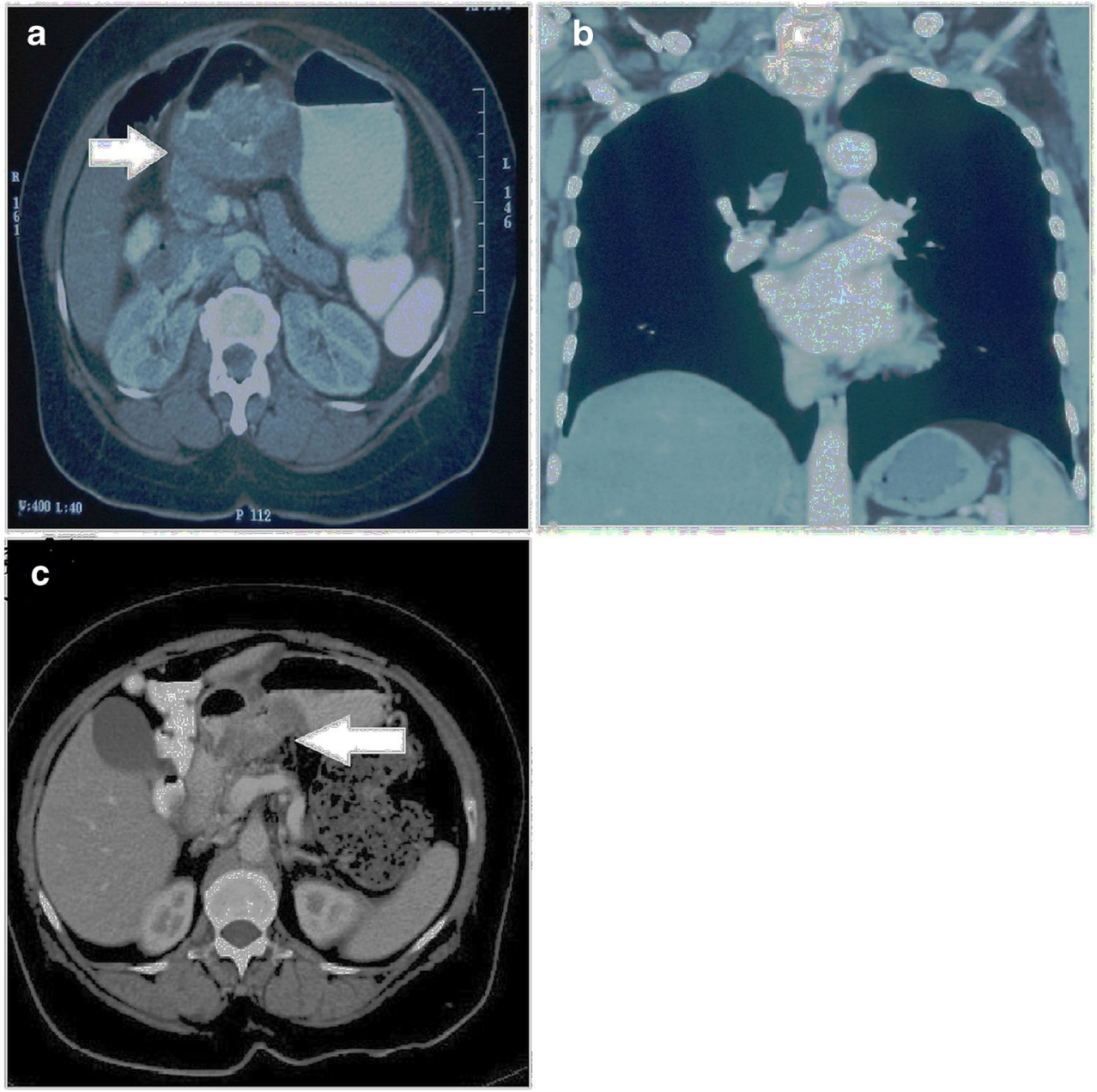

theory behind its origin is retro differentiation of adenocarcinoma into choriocarcinoma because $70 \%$ of cases show adenocarcinoma component along with it [3-5]. Pure choriocarcinoma of stomach as in our case is seen only in $30 \%$ of cases [7]. Other hypotheses proposed are development from putative displaced gonadal anlage or due to the delayed metastasis of unidentified primary lesion in the uterus. Some also support its origin from a Teratoma [8].

Diagnosis is usually made by clinical examination, estimation of serum $\beta$-HCG levels, radiological tests and histological findings. It is difficult to differentiate between adenocarcinoma and choriocarcinoma. Upper GI endoscopy and biopsy is also useful but it is often difficult to obtain sufficient material for confirmation and only 8 to $15 \%$ of cases are diagnosed by endoscopic biopsy accurately as in our case [7].
Choriocarcinoma is usually exophytic with beefy red, hemorrhagic appearance which shows increased vascularity. For pathological diagnosis, assessment of cytotrophoblast and syncytiotrophoblast are prerequisite, and it can be confirmed by $\beta$-HCG positive cells in immunohistochemical test and high elevation of $\beta-\mathrm{HCG}$ in the blood [9]. Other histological features are intratumoral haemorrhage, necrosis, and vascular invasion. Primary choriocarcinoma in stomach is well established by absence of tumour in ovaries, testis, retroperitoneum etc. We ruled out primary in gonads and other sites by extensive preoperative evaluation.

Management of gastric choriocarcinoma is indistinct. The most recent literature supports surgery as the primary modality of treatment. PGC does not respond well to chemotherapy as is seen with gonadal choriocarcinoma. Preferred treatment includes gastrectomy 
Fig. 3 a, b. Haematoxylin and eosin staining of tumour.

Histopathology pictures of the tumour showing bizarre gaint cells (arrow) which are multi nucleated or lobulated nucleus (syncytiotrophoblasts) with oval or polygonal cells composed of clear or pale cytoplasm (Cytotrophoblasts) suggestive of choriocarcinoma of stomach. c. Immunohistochemical staining picture of the tumour showing strong positivity to $\beta-\mathrm{HCG}$ (arrow)., b. Haematoxylin and eosin staining of tumor. Histopathology pictures of the tumour showing bizarre gaint cells (arrow) which are multi nucleated or lobulated nucleus (syncytiotrophoblasts) with oval or polygonal cells composed of clear or pale cytoplasm

(Cytotrophoblasts) suggestive of choriocarcinoma of stomach. c. Immunohistochemical staining picture of the tumour showing strong positivity to $\beta-\mathrm{HCG}$ (arrow)

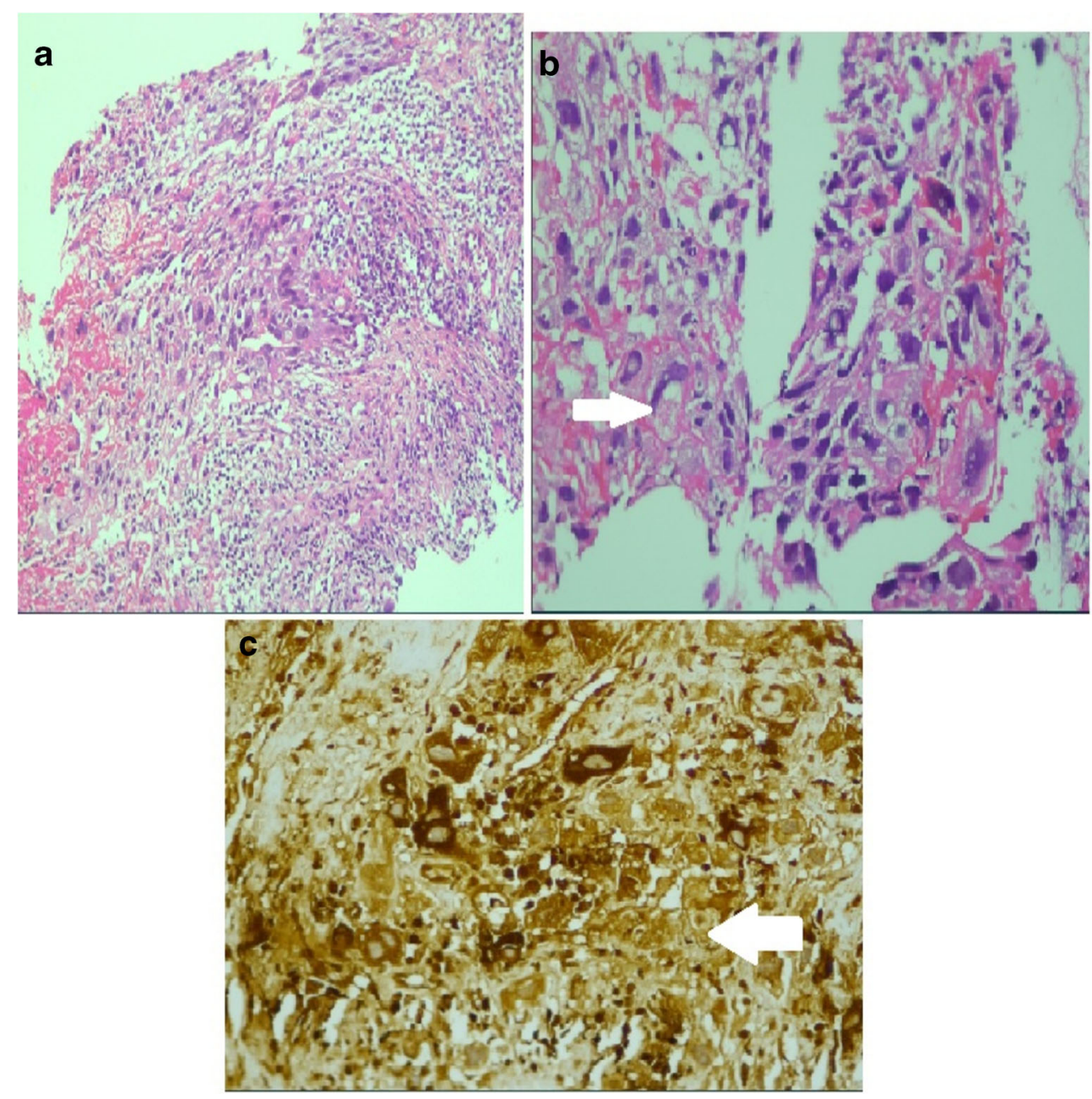

with lymph node dissection followed by chemotherapy. Metastatic PGC can be treated by chemotherapy. Commonly used chemotherapeutic agents include Methotrexate, Actinomycin D, Etoposide, Folinic acid, Vincristine, Cyclophosphamide etc. [1, 2, 10-12]. Palliative procedures like partial gastrectomy can be done in patients with upper gastrointestinal haemorrhage. Kazuhiro Takahashi et al. reported usefulness of computed tomography-guided radio frequency ablation (RFA) in combination with surgery and chemotherapy for oligometastatic tumour and longest survival of 10 years for their patient with this multimodality of treatment [13]. For choriocarcinoma and adenocarcinoma, chemotherapy modality is different hence in cases of adenocarcinoma with elevated serum $\beta$-HCG, assessing presence of choriocarcinomatous component is a must.

Prognosis is very poor and mean survival time of patients with PGC is less than 6 months. In our case survival period was only 5 months. The most common cause of death is liver failure secondary to metastasis [6].
To conclude, even though primary choriocarcinoma of stomach is very rare, it should be kept in differentials when evaluating gastric neoplasms. Diagnosis can be made only by histopathology and immunohistochemistry. Recent literature supports surgery as the primary modality of treatment. Early diagnosis of PGC gives best outcome.

\section{References}

1. Liu Z, Mira JL, Cruz-Caudillo JC (2001) Primary gastric choriocarcinoma: a case report and review of the literature. Arch Pathol Lab Med 125(12):1601

2. Noguchi T, Takeno S, Sato T, Takahashi Y, Uchida Y, Yokoyama S (2002) A patient with primary gastric choriocarcinoma who received a correct preoperative diagnosis and achieved prolonged surgery. Gastric Cancer 5(2):112-7.3.3

3. Wurzel J, Brooks JJ (1981) Primary gastric choriocarcinoma: immunohistochemistry, postmortem documentation, and hormonal effects in a postmenopausal female. Cancer 48:2756-2761 
4. Garcia RL, Ghali VS (1985) Gastric choriocarcinoma and yolk sac tumor in a man: observations about its possible origin. Hum Pathol 16:955-958

5. Imai I, Kawabe T, Takahashi M et al (1994) A case of primary gastric choriocarcinoma and a review of the Japanese literature. J Gastroenterol 29:642-646

6. Kobayashi A, Hasebe T, Endo Y et al (2005) Primary gastric choriocarcinoma: two case reports and a pooled analysis of 53 cases. Gastric Cancer 8(3):178-185

7. Kırkıl C, Karabulut K, Aygen E, İlhan YS, Mesut Y, Özercan İH (2012) A case of primary gastric choriocarcinoma and a review of the Turkish literature: an extremely rare carcinoma of the stomach. Dicle Med J 39(1):105-109

8. Hartz PH, Ramirez CA (1953) Coexistence of carcinoma and chorioepithelioma in the stomach of a young man. Cancer 6:319326
9. Yoon JH, Kim MS, Kook E0H, Ahn SH, Jeong SY, Han MS et al (2008) Primary gastric choriocarcinoma: two case reports and review of the literatures. Cancer Res Treat 40(3):145-150

10. Jindrak K, Bochetto JF, Alpert LI (1976) Primary gastric choriocarcinoma: case report with review of world literature. Hum Pathol 7: 595-604

11. Manabe T, Adachi M, Hirao K (1885) Human chorionic gonadotropin in normal, inflammatory, and carcinomatous gastric tissue. Gastroenterology 89:1319-1325

12. Anjaneyulu, Rao SC, Rao RV (2000) Primary choriocarcinoma of stomach. Indian J Pathol Microbiol 43:471-474

13. Takahashi K, Tsukamoto S, Saito K, Ohkohchi N, Hirayama K (2013) Complete response to multidisciplinary therapy in a patient with primary gastric choriocarcinoma. World J Gastroenterol 19(31):5187-5194 